\title{
Death due to intestinal obstruction in a patient treated with clozapine
}

\author{
Nilukshi Abeyasinghe, Tikiri Bandara Gunathilake, Harischandra Gambheera
}

\section{Summary}

A 53 year old female diagnosed with long term resistant schizophrenia, ischaemic heart disease and hypertension was treated with $700 \mathrm{mg} /$ day of clozapine. Five months after commencing treatment, she complained of acute onset colicky abdominal pain and nausea with constipation of one days duration. Suspecting intestinal obstruction, she was referred from a psychiatry unit to the casualty ward. On examination, she had a distended abdomen and tenderness. No abdominal masses were detected. A stool softener and lactulose was given. However, five hours later, she died. Autopsy revealed a grossly distended intestine extending from the stomach to the rectum. There were solid and liquid faeculent material commencing from the oesophagus through to the rectum. No mechanical obstruction was evident. A sector of the ileum showed an area of patchy haemorrhage. Histology showed villous atrophy with flattened epithelial cells and fibrosis of the lamina propria. The heart had a few scattered areas of fibrosis. Other macroscopic and microscopic findings were unremarkable. The cause of death was recorded as intestinal obstruction probably induced by clozapine. The literature has reported a prevalence of constipation associated with clozapine ranging from $14-60 \%$. Higher dose schedules and prolonged treatment are contributory factors. Deaths from clozapine have been reported due to intestinal obstruction, aspiration of faeculent matter, perforation and peritonitis and following surgery to decompress the bowel.

\section{SL J Psychiatry 2010; 1 (2): 64-66}

\section{Introduction}

Clozapine is used in the treatment of resistant schizophrenia. The important side-effects usually associated with clozapine treatment include postural hypotension, weight gain, tachycardia, cardiomyopathy, cardiomyositis, seizures, hypersalivation and agranulocytosis. Constipation has been reported as a side effect in many clinical trials. The Sandoz (Sandoz Pharmaceutical Corporation, East Hanover, NJ) package insert records a $14 \%$ prevalence (1). Lieberman et al noted constipation with clozapine in $33.3 \%$ during acute treatment and $22.8 \%$ during maintenance treatment (2). Hayes and Gibler found a $60 \%$ prevalence of constipation in their evaluation of clozapine (1). This effect is believed to occur due to its anticholinergic effects. Although the presentation of constipation may be insignificant and of short duration as was in this case report, it could lead to death.

Levin, Barrett and Mendelowitz reported a death in a patient receiving clozapine $(750 \mathrm{mg} /$ day $)$ for the past six years, who had a gastroscopy six months before his admission for investigation of vomiting and epigastric pain (3). On admission with an acute abdomen, emergency laparotomy was done to reveal large bowel obstruction by faecal impaction. Total colectomy and ileostomy were performed. However death occurred three weeks later due to septic shock and multisystem organ failure. Drew, Herdson, Hayes and Gibler reported of two patients on clozapine $500 \mathrm{mg} /$ day for two years and $400 \mathrm{mg} /$ day for thirty six days respectively who died as a consequence of inhalation of faeculent vomitus subsequent to constipation and bowel obstruction $(1,4)$.
Freudenreich and Goff document a patient on clozapine $400 \mathrm{mg}$ /day for six weeks who developed perforation of the colon and peritonitis requiring hemicolectomy who also sustained perioperatively a dense cerebrovascular accident (5). Shammi and Remington describe a death where a patient on clozapine $600 \mathrm{mg} /$ day for four months presented with constipation and an acute abdomen. Laporotomy revealed a grossly dilated colon (6). Autopsy revealed evidence of necrosis and dilation of the entire large intestine.

This case report is of a death in a patient who developed fatal intestinal obstruction while on treatment with clozapine.

\section{Case Report}

A 53 year old female diagnosed with resistant schizophrenia for thirty two years was treated with clozapine for five and a half months. She was on a dose of $700 \mathrm{mg} /$ day at the time of her death. She was also taking sodium valproate $600 \mathrm{mg} / \mathrm{day}$. One year ago she had complained of chest pain. An electrocardiogram (ECG) revealed Left Bundle Branch Block and she was also diagnosed with hypertension. She was treated with captopril $(25 \mathrm{mg} /$ day $)$, isosorbide dinitrate (30 mg/day) and atorvastatin ( $20 \mathrm{mg}$ /day). The white blood cell counts were regularly monitored. She presented with sudden onset colicky abdominal pain and nausea. She complained of constipation of one days duration. Suspecting intestinal obstruction, she was transferred from a psychiatry unit to the casualty ward. On admission, she was drowsy and dehydrated. 
Her abdomen was distended with tenderness and there were increased bowel sounds. No abdominal masses were detected. A stool softener and lactulose was given as initial therapy. However, five hours later, she died.

\section{Autopsy}

Autopsy revealed gross distension of the intestine extending from the stomach to the rectum. There were solid and liquid faeculent material within the oesophagus and extending through to the rectum. No mechanical obstruction was evident. However, there were areas of solid faecal matter within the colon which was grossly dilated and pale. A sector of the ileum showed an area of patchy haemorrhage in the serosa. Histology in the ileum showed villous atrophy with flattened epithelial cells and fibrosis of the lamina propria. The colonic mucosa did not show evidence of inflammation, ischaemic necrosis, pseudomembranes or vascular thrombi. The heart had a few scattered areas of fibrosis. Other macroscopic and microscopic findings were unremarkable.

The cause of death was recorded as intestinal obstruction with evidence of oesophageal reflux probably induced by clozapine.

\section{Discussion}

Constipation is a relatively common side effect of clozapine (7). This may be commonly ignored because of the importance of treating resistant schizophrenia resulting in a delay in diagnosis and progress of the intestinal obstruction with fatal consequences. The acute onset of colicky abdominal pain with distension and tenderness may also be masked in patients with schizophrenia as a result of their altered sensitivity to pain and possible difficulty in expressing that pain $(8,9,10)$. Faecal stasis occurs as a result of inhibition of intestinal motility due to the anticholinergic properties of clozapine (11). Gastric outlet obstruction and a subileal condition have also been reported $(12,13)$. This phenomenon was very evident in this patient who

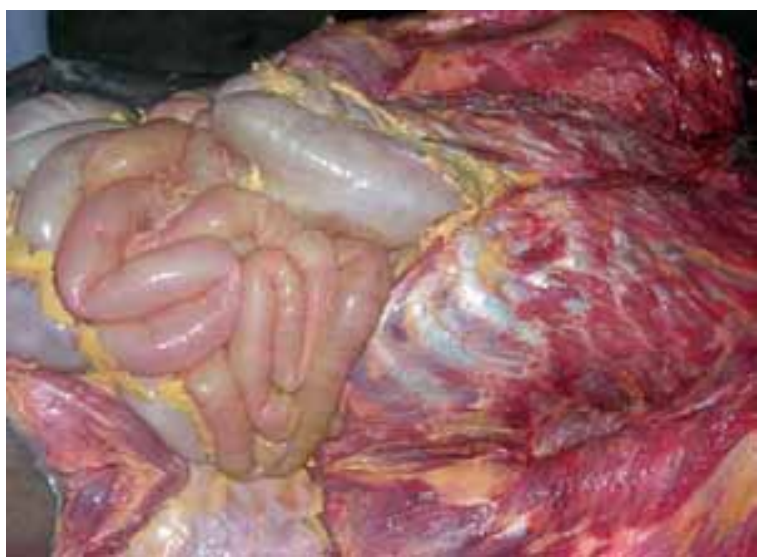

Fig. 1 Grossly distended intestine on opening the abdomen

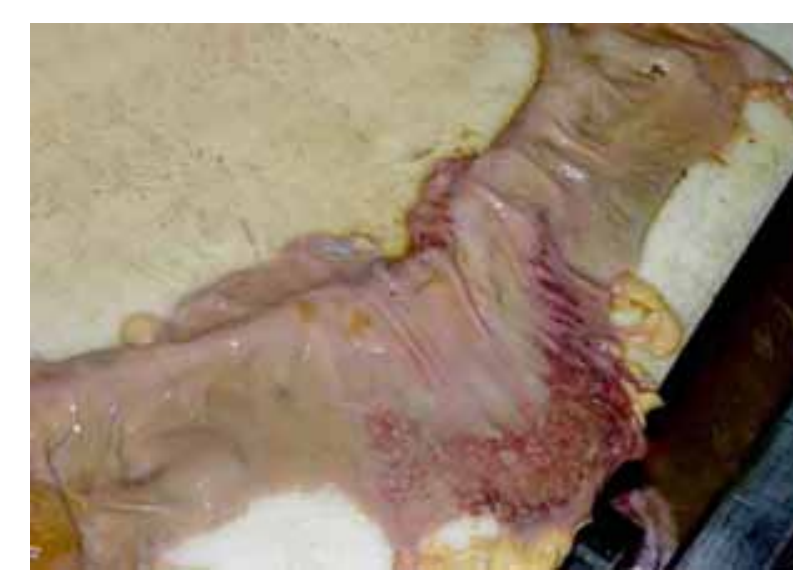

had faeculent fluid extending upto the oesphagus with evidence of oesophageal reflux. Drew and Herdson noted similar features in their patient and also recorded a complaint of constipation only once, eleven days before his death (4). Shammi and Remington noted a focal area with haemorrhagic adhesions of the serosa in the distal ileum as was seen in this patient (6). The most marked changes they documented were in the dilated colon which histologically, showed evidence of necrotizing colitis. In this patient the visible changes were those of villous atrophy, flattened epithelial cells and fibrosis of the lamina propria of the ileum with a few scattered lymphocytes which to our knowledge has not been documented before. Pelizza and Melegaril first reported a case of clozapine-induced microscopic colitis in which they report an intraepithelial lymphocytosis more predominant in the surface epithelium and a lymphocyte predominant mixed inflammatory infiltrate in the lamina propria, an architecturally preserved colonic mucosa and a subepithelial collagen band (14). Karmacharya, Mino and Pirl reported evidence of an eosinophilic colitis showing patchy eosinophilic infiltrate and histiocytic aggregates focally associated with crypt destruction in an architecturally preserved colonic mucosa (15). The presence of colitis could occur due to the anticholinergic activity of clozapine either from faecal stasis and dilatation, causing microbial proliferation or by compromise of the vascular supply as a result of colonic distension (6). However case reports without colitis as was seen in this patient have also been reported (16). A syndrome of clozapineinduced gastrointestinal hypomotility was described in 2008 after analysis of 102 cases with an estimated Risk factors included high doses of clozapine (the mean dose being $428 \mathrm{mg} /$ day), use of other anticholinergic medications and a history of gastrointestinal disease and bowel surgery among other factors. This patient was on a dose of $700 \mathrm{mg} /$ day for five and a half months and was also on valproate which could cause a minor increase in clozapine metabolites. In the reported cases, $20 \%$ developed the syndrome within one month of start of treatment, $36 \%$ within 4 months and more than $50 \%$ within the first year.

Fig 2 Area of patchy haemorrhage in the ileum prevalence of $0.3 \%$ and a mortality rate of $27.5 \%(17)$. 
Responses to some of these deaths include a clozapine constipation protocol (1). An abdominal physical assessment and a daily flow sheet documenting bowel and dietary habits together with use of exercise, fluids and a high-fibre diet, a bulk-forming laxative or stool softener were initiated. A further change included a slower clozapine titration, increasing doses by no more than $25 \mathrm{mg} /$ day to a maximum of $100 \mathrm{mg} / \mathrm{week}$, and treatment for some patients with bethanechol to prevent clozapine-induced constipation. These remedies seem to have decreased the prevalence of constipation in the population studied, with fewer aggressive interventions being required. Ideally facilities to monitor the serum level of clozapine would indicate whether the dose could be lowered or whether it is insufficient. A serum level of less than $350 \mu \mathrm{g} / \mathrm{l}$ results in poor clinical response, while levels in the range of $500-700 \mu \mathrm{g} / \mathrm{l}$ or above could be considered for dose reduction (18).

This case highlights the morbidity and mortality associated with clozapine-induced gastrointestinal hypomotility and the importance of screening such patients. Presentation with an acute abdomen in a patient on clozapine therapy with a history of constipation should be considered a medical emergency.

\section{Declaration of interest}

None

Nilukshi Abeyasinghe, Senior Lecturer in Forensic Medicine, Tikiri Bandara Gunathilake, Senior Registrar in Forensic Medicine, Department of Forensic Medicine and Toxicology, Faculty of Medicine, University of Colombo, Sri Lanka.

Harischandra Gambheera, Consultant Psychiatrist, National Institute of Mental Health, Angoda, Sri Lanka Corresponding author

Nilukshi Abeyasinghe, Department of Forensic Medicine and Toxicology, Faculty of Medicine, 25 Kynsey Road, Colombo 8, Sri Lanka.

Email: abeynl@yahoo.co.uk

\section{References}

1. Hayes G, Gibler B. Clozapine -Induced Constipation (letter). Am J Psychiatry 1995; 12:152

2. Lieberman MD, Safferman AZ, Pollack S et al. Clinical effects of Clozapine in chronic schizophrenia:response to treatment and predictors of outcome. Am J Psychiatry $1994 ; 151: 1744-52$
3. Levin TT, Barrett J, Mendlowitz AM. Death from Clozapine-Induced Constipation. Psychosomatics 2002;43(1):71-3

4. Drew L, Herdson P. Clozapine and constipation: a serious issue Aust NZ J Psychiatry 1997;31:149-50.

5. Freudenreich O, Goff DC. Colon perforation and peritonitis associated with Clozapine. J Clin Psychiatry 2000;61:950-1.

6. Shammi CM, Remington G: Clozapine induced necrotizing colitis. J Clin Psychopharmacol 1997;17:230-32.

7. John JP, Chengappa KNR, Baker RW et al. Assessment of changes in weight and frequency of use of medication for the treatment of gastrointestinal symptoms among clozapine-treated patients. Ann Clin Psychiatry 1995;7:119-25.

8. Rosenthal SH, Porter KA, Coffey B. Pain insensitivity in schizophrenia. Case report and review of the literature. Gen Hosp Psychiatry 1990;12:319-22

9. Bender L, Schilder P. Unconditioned and conditioned reactions to pain in schizophrenia. Am J Psychiatry 1930;3:365-84

10. Bickerstaff LK, Harris SC, Leggett RS et al. Pain insensitivity in schizophrenic patients. A surgical dilemma. Arch Surg 1988;123: 49-51.

11. Coward DM. General pharmacology of clozapine. Br J Psychiatry1992;160[suppl 17]:5-11.

12. Schwartz BJ, Frisolone JA. A case report of clozapineinduced gastric outlet obstruction [letter]. Am J Psychiatry 1993;150:1563.

13. Fitton A, Heel RC. Clozapine: a review of its pharmacological properties, and therapeutic use in schizophrenia. Drugs 1990;40(5):722-47.

14. Pelizza L, Melegari M. Clozapine-induced microscopic colitis: A case report and review of the literature. J Clin Psychopharmacol 2007;27(6):571-74.

15. Karmacharya R, Mino M, Pirl WF. Clozapine-induced eosinophilic colitis. Am J Psychiatry 2005;162:138687.

16. Alam HB, Fricchione GL, Guimaraes ASR, Zukerberg LR. A 26 year old man with abdominal distension and shock. N Engl J Med 2009; 361:1487-96

17. Palmer SE, McLean RM, Ellis PM, Harrison-Woolrych M. Life-threatening clozapine- induced gastrointestinal hypomotility: an analysis of 102 cases. J Clin Psychiatry 2008; 69(5):759-68.

18. Kronig MH, Munne RA, Szymanski S et al. Plasma clozapine levels and clinical response for treatmentrefractory schizophrenic patients. Am J Psychiatry 1995;152:179-82. 